\title{
Bioprinting neural tissues using stem cells as a tool for screening drug targets for Alzheimer's disease
}

\author{
Stephanie M Willerth*,1,2 \\ ${ }^{1}$ Department of Mechanical Engineering \& Division of Medical Sciences, University of Victoria, Victoria, BC V8W 2Y2, Canada \\ ${ }^{2}$ International Collaboration on Repair Discoveries, University of British Columbia, Vancouver, BC V5Z 1M9, Canada \\ *Author for correspondence: Tel.: +250 721 7303; Fax: +250 721 6051; willerth@uvic.ca
}

First draft submitted: 13 July 2017; Accepted for publication: 19 October 2018; Published online: 30 November 2018

Keywords: bioinks • biomaterials • dementia • disease models • neuroscience $\bullet$ tissue engineering

\section{Current status of identifying potential drug targets for Alzheimer's disease}

Identifying and validating effective drugs for treating Alzheimer's disease, the most common form of dementia, remains a challenging and expensive process. The prevalence of Alzheimer's disease increases in the aging population with other environmental factors contributing to likelihood of disease. The symptoms of Alzheimer's disease consist of a progressive decline in cognitive function, including an inability to remember new information and decreased language and reasoning function. The pathology of Alzheimer's disease includes the presence of plaques containing aggregates of amyloid beta $(\alpha \beta)$ proteins and tangles containing neurofibrillary tangles. Certain genetic mutations increase the possibility of developing Alzheimer's disease [1]. These mutations consist of an altered APP gene responsible for encoding amyloid precursor protein, along with the PSEN1 and PSEN2 mutations cause decreased activity by the $\gamma$-secretase complex. These mutations result in improper processing of the $\alpha \beta$ proteins. Currently, approved treatments for Alzheimer's disease include four different types of cholinesterase inhibitors and the drug memantine - a $N$-methyl-D-aspartate receptor antagonist that targets glutaminergic neurons to preserve their function [2]. However, one of these five drugs (tacrine) is no longer prescribed due to issues with liver toxicity. Memantine has been the only compound approved for treating Alzheimer's disease since 2000 even though more than 200 potential compounds being evaluated in over 400 clinical trials [3]. Additionally, its efficacy as a treatment for Alzheimer's disease remains controversial. These issues with toxicity and efficacy of potential drug targets suggest that the current tools used for preclinical drug screening to identify target compounds are ineffective and must be improved to enable better selection of potential drug targets before they reach the stage of clinical trials. Such tools would lead to a decrease in the significant costs associated with drug development, accelerating the process of finding effective treatments. Current methods for evaluating target compounds consist of animal models of disease and the use of cadaveric human tissues. In addition to their lack of predictive capacity, animal models are costly, and the supplies of human neural tissues remain limited. Developing more effective assays for preclinical identification of drug targets will lower the expense of the drug discovery process and increase the likelihood of successful outcomes at the stage of clinical trials.

\section{Stem cells as a powerful tool for tissue engineering \& drug discovery}

The discovery of human induced pluripotent stem cells (hiPSCs) in 2007 opened new doors for regenerative medicine and tissue engineering [4]. Scientists demonstrated that somatic cells, like fibroblasts, could be reprogrammed using transcription factors back into a pluripotent state where they could produce any type of tissue present in the human body, including neural tissues. This technology generated new avenues for studying neurodegenerative diseases with a genetic component like Alzheimer's disease. Cells taken from patients suffering from Alzheimer's disease could be reprogrammed into hiPSC lines, which could then serve as a tool for understanding how the disease progresses as well as for screening potential drug targets in vitro [5]. The use of hiPSC lines containing the different genetic mutations associated with Alzheimer's disease also enables the use of personalized medicine for treating different subsets of the disease. While cells are often cultured on 2D substrates in vitro, these conditions 
do not accurately mimic the microenvironment present in the CNS. A subset of the field of regenerative medicine known as tissue engineering generates systems for producing functional tissues through combing cells, biomaterials and drug delivery. A range of different combinations of cells, biomaterials and drug delivery systems have been evaluated as ways to engineer neural tissues both in vitro and in vivo [6]. Recent efforts have focused on adapting these principles of tissue engineering to hiPSCs to produce functional neural tissues for a variety of applications, including as a tool for drug screening. For example, a group from the University of Wisconsin-Madison demonstrated how $3 \mathrm{D}$ tissues produced from human embryonic stem cells could predict compound toxicity [7]. An alternative tissue engineering strategy harnesses the power of pluripotent stem cells to recapitulate the events of development to generate structures known as organoids, mini tissues containing the features of the organ being reproduced such as the brain [8]. More recently, researchers have generated brain organoids from hiPSC lines containing the PSEN mutations associated with Alzheimer's disease and confirmed they respond in a physiologically relevant manner to different treatment conditions [9]. This work suggests the potential of brain organoids as an important tool for drug screening as well. However, these engineered tissues and brain organoids require a significant amount of labor to generate physiologically relevant structures.

\section{The role of 3D printing in automating the tissue engineering process}

3D bioprinting can provide a high-throughput, reproducible alternative to these aforementioned methods for engineering neural tissues [10]. While some of the more commonly used 3D printing methods, like fused deposition modeling, selective laser sintering and stereolithography, can produce scaffolds capable of supporting pluripotent stem cell culture, it remains difficult to control the precise location when seeding multiple types of cells or localizing specific cues for controlling stem cell behavior. 3D bioplotting and microfluidic 3D printing methods allow for mixing of cells with bioinks, materials that can be printed while supporting cell culture, enabling the production of neural tissues. Pluripotent stem cells and their derivatives require specific culture conditions to ensure their viability and differentiation state and thus are often more difficult to 3D print into the desired structures when engineering neural tissue. Recent advances in bioprinting have enabled the printing of hiPSCs using microfluidic extrusion, opening the possibility for applying this technology for high-throughput production of hiPSC-derived neural tissues [11]. Recent developments in 3D bioprinting technologies make printing physiologically relevant neural tissues derived from hiPSCs a real possibility. These developments include advances in both the methods used for $3 \mathrm{D}$ printing and the development of multifunctional bioinks. For example, Aspect Biosystems currently markets a novel bioprinter called the RX1, which uses Lab-on-a-printer ${ }^{\mathrm{TM}}$ technology to print a variety of materials and cell types for generating living tissues on demand [12,13]. Additionally, a novel 3D printing method was developed at Carnegie Mellon that uses freeform reversible embedding of suspended hydrogels to generate complex structures found in the body [14]. Such hydrogels could be produced for supporting hiPSC culture and differentiation into the structures found in the brain. These hydrogels can be functionalized with cues like laminin to enhance cell survival and differentiation [15]. Other potential additives to bioinks include morphogen releasing microspheres for directing hiPSCs to form neural tissues. My group has successfully developed microspheres for delivery of two small molecule morphogens - guggulsterone and purmorphamine [16,17]. Our data have shown that such microspheres are powerful tools for promoting the differentiation of hiPSCs into neural tissues and being able to place different combinations and concentrations of microspheres into precise locations using $3 \mathrm{D}$ printing would enable production of tissues similar to that found in the brain and spinal cord. These 3D-printed neural tissues could then be used for screening potential drug targets for Alzheimer's disease as an alternative to expensive preclinical animal testing.

\section{Avenues for future work}

Future work must address certain issues to produce physiologically relevant, functional neural tissues. Areas for future development of this technology include incorporation of a stable vasculature, so larger constructs can be generated as well as for mimicking the blood-brain barrier found in vivo. These neural tissues could be printed in arrays when screening target drugs. Such work could also contribute to development of engineered tissues that could be transplanted for regenerating damaged regions of the central nervous system. Overall, 3D bioprinting hiPSC-derived neural tissues hold significant potential as a novel way to generate new tools for screening drug targets for the treatment of Alzheimer's disease. 
Financial \& competing interests disclosure

SM Willerth receives funding from the Canada Research Chairs program, the Natural Science and Engineering Research Council, and the British Columbia Innovation Council's Ignite Program to support this work. SM Willerth also has a commercialization agreement with Aspect Biosystems with regards to 3D printing neural tissues and a provisional patent on a bioink.

No writing assistance was utilized in the production of this manuscript.

\section{Open access}

This work is licensed under the Attribution-NonCommercial-NoDerivatives 4.0 Unported License. To view a copy of this license, visit http://creativecommons.org/licenses/by-nc-nd/4.0/

\section{References}

1. Jarmolowicz AI, Chen H-Y, Panegyres PK. The patterns of inheritance in early-onset dementia: Alzheimer's disease and frontotemporal dementia. Am. J. Alzheimers Dis. Other Demen. 30(3), 299-306 (2015).

2. Anand R, Gill KD, Mahdi AA. Therapeutics of Alzheimer's disease: past, present and future. Neuropharmacology 76, 27-50 (2014).

3. Parsons CG, Stöffler A, Danysz W. Memantine: a NMDA receptor antagonist that improves memory by restoration of homeostasis in the glutamatergic system-too little activation is bad, too much is even worse. Neuropharmacology 53(6), 699-723 (2007).

4. Takahashi $\mathrm{K}$, Tanabe $\mathrm{K}$, Ohnuki $\mathrm{M}$ et al. Induction of pluripotent stem cells from adult human fibroblasts by defined factors. Cell 131(5), 861-872 (2007).

5. Arber C, Lovejoy C, Wray S. Stem cell models of Alzheimer's disease: progress and challenges. Alzheimers Res. Ther. 9(1), 42 (2017).

6. Willerth S. Engineering Neural Tissue from Stem Cells. Academic Press, MA, USA (2017).

7. Schwartz MP, Hou Z, Propson NE et al. Human pluripotent stem cell-derived neural constructs for predicting neural toxicity. Proc. Natl Acad. Sci. 112(40), 12516-12521 (2015).

8. Di Lullo E, Kriegstein AR. The use of brain organoids to investigate neural development and disease. Nat. Rev. Neurosci. 18(10), 573 (2017).

9. Yan Y, Song L, Bejoy J et al. Modeling neurodegenerative microenvironment using cortical organoids derived from human stem cells. Tissue Eng. Part A. 24(13-14), 1125-1137 (2018).

10. Thomas M, Willerth SM. 3-D bioprinting of neural tissue for applications in cell therapy and drug screening. Front. Bioeng. Biotechnol. 5, 69 (2017).

11. Gu Q, Tomaskovic-Crook E, Wallace GG, Crook JM. 3D bioprinting human induced pluripotent stem cell constructs for in situ cell proliferation and successive multilineage differentiation. Adv. Healthc. Mater. 6(17), doi:10.1002/adhm.201700175 (2017) (Epub ahead of print).

12. Beyer ST, Walus K, Mohamed T, Bsoul AAM. System for additive manufacturing of three-dimensional structures and method for same. US Patent 20,160,136,895 (2016).

13. Bsoul A, Pan S, Cretu E, Stoeber B, Walus K. Design, microfabrication, and characterization of a moulded PDMS/SU-8 inkjet dispenser for a Lab-on-a-Printer platform technology with disposable microfluidic chip. Lab Chip 16(17), 3351-3361 (2016).

14. Hinton TJ, Jallerat Q, Palchesko RN et al. Three-dimensional printing of complex biological structures by freeform reversible embedding of suspended hydrogels. Sci. Adv. 1(9), e1500758 (2015).

15. Suri S, Han L-H, Zhang W, Singh A, Chen S, Schmidt C. Solid freeform fabrication of designer scaffolds of hyaluronic acid for nerve tissue engineering. Biomed. Microdevices 13(6), 983-993 (2011).

16. Agbay A, De La Vega L, Nixon G, Willerth SM. Guggulsterone-releasing microspheres direct the differentiation of human induced pluripotent stem cells into neural phenotypes. Biomed. Mater. 13(3), 034104 (2018).

17. de La Vega L, Karminin K, Willerth S. Engineering neural tissue from human pluripotent stem cells using novel small molecule releasing microspheres. Adv. Biosyst. doi:10.1002/adbi.201800133 (2018) (Epub ahead of print). 
